ESJ Social Sciences

\title{
Transition à la Parentalité et Fonctionnement Conjugal chez les Couples Infertiles en Tunisie
}

\author{
Najoua Ghrir \\ Docteur en psychologie, Psychologue Principale et sous directeur au \\ Ministère des Affaires Sociales, Tunisie
}

Doi:10.19044/esj.2021.v17n41p84

Submitted: 23 November 2021

Accepted: 06 December 2021

Published: 31 December 2021
Copyright 2021 Author(s)

Under Creative Commons BY-NC-ND

4.0 OPEN ACCESS

Cite As:

Ghrir N. (2021). Transition à la Parentalité et Fonctionnement Conjugal chez les Couples Infertiles en Tunisie. European Scientific Journal, ESJ, 17 (41), 84.

https://doi.org/10.19044/esj.2021.v17n41p84

\section{Résumé}

Objectif.-Evaluer l'impact de l'adoption sur le fonctionnement conjugal des couples infertiles. Méthode.-L'échantillon est composé de 146 participants hétérosexuels (73 couples) répartis en un groupe avec enfant adoptif (GAEA) composé de 30 couples infertiles vivant une parentalité adoptive et deux groupes contrôles ; le groupe avec enfant biologique (GAEB) composé de 30 couples fertiles ayant accès à la parentalité biologique et le groupe sans enfant (GSE) qui comprend 13 couples infertiles sans enfants. Résultats : Le GAEA semble éviter moins la proximité que les deux groupes contrôles et apparaît plus anxieux face à l'abandon uniquement par rapport au GAEB. Il utilise significativement plus la communication mutuelle mais uniquement par rapport au GSE et rapporte davantage des comportements de "Demande-Retrait" par rapport aux deux groupes témoins. De plus, Il est plus satisfait sur le plan conjugal que les deux groupes contrôles. Conclusion: L'étude offre une meilleure compréhension des changements au sein des couples infertiles devenant parents par voie d'adoption et ouvre plusieurs pistes d'investigation.

Mots-clés: Parentalité adoptive, évènements stressants, attachement, communication, ajustement dyadique 


\title{
Transition to Parenthood and Marital Functioning Among Infertile Couples in Tunisia
}

\author{
Najoua Ghrir \\ Docteur en psychologie, Psychologue Principale et sous directeur au \\ Ministère des Affaires Sociales, Tunisie
}

\begin{abstract}
The aim of this study is to assess the impact of adoption on the marital functioning of infertile couples. The sample is composed of 146 heterosexual participants (73 couples) divided into a group with adopted children (GWAC) made up of 30 infertile couples living in adoptive parenthood and two control groups; the group with biological child (GWBC) made up of 30 fertile couples with access to biological parenthood and the childless group (GWC) which includes 13 infertile couples without children. The GWAC seems to avoid proximity less than the two control groups and appears more anxious about abandonment only in relation to the GWBC. He used mutual communication significantly more but only in relation to the GWC and reported more "Request-Withdrawal" behaviors compared to the two control groups. In addition, he is more marital satisfaction than the two control groups. The study offers a better understanding of the changes in infertile couples becoming parents by adoption and opens several tracks of investigation.
\end{abstract}

Keywords: Adoptive parenting, stressful events, attachment, communication, dyadic adjustment

\section{Introduction}

La transition à la parentalité est un évènement très important « voire un moment charnière dans la vie d'un couple » (Zeghiche, \& deMontigny, 2014, p3). En Tunisie, les données empiriques quant aux effets de la transition à la parentalité sur la relation conjugale sont presque absentes plus particulièrement concernant l'adoption malgré la grande importance des pratiques adoptives par les couples infertiles.

En Tunisie comme dans tout le Maghreb, l'adoption d'enfants est un sujet «étonnamment peu investi » et les quelques sources scientifique se limitent en «une modeste production de juristes, de quelques historiens, psychologues et sociologues et d'une seule anthropologue » (Barraud, 2008, p.2). La majorité des recherches s'est plutôt intéressée à la qualité de la relation conjugale lors de la transition à la parentalité chez des couples capables de procréer ou chez des couples infertiles mais qui ont réussi à devenir parents grâce à une procréation médicalement assistée. L'objectif de la présente étude 
est d'examiner la parentalité adoptive et ses effets sur différentes dimensions de la relation conjugale chez les couples tunisiens.

\section{Transition à la parentalité}

La transition à la parentalité est considérée comme une crise parce qu'elle amène le couple à modifier sa relation pour englober un système de trois personnes, à accepter qu'une relation co-parentale s'ajoute à sa relation conjugale et faire des réajustements pour garder l'équilibre de cette dernière (Frascarol, Darwiche, \& Favez, 2009).

La période suivant la naissance de l'enfant chez les parents biologiques et celle de l'élaboration du processus de parentalité chez les parents adoptifs deviennent des événements stressants (Vinay, Brenot-Bergeret, Rosenblum, $\&$ Genty, 2014). Chez ces derniers, l'adoption est, dans la plupart du temps, la conséquence d'un autre évènement stressant qui est l'infertilité. Cependant, ces deux évènements demeurent de nature différente. L'infertilité est un évènement négatif (triste) brusque et inattendu, et en lien avec un problème de santé, alors que l'adoption, étant une solution à ce problème, constitue un évènement positif (heureux) survenant par le propre choix du couple et produisant des changements dans sa vie conjugale et influençant son fonctionnement et sa stabilité.

Le modèle de fonctionnement du couple Vulnérabilité-StressAdaptation (VSA) développé par Karney et Bradbury (1995) peut être utilisé pour vérifier et expliquer le fonctionnement conjugal lors de la transition à la parentalité, puisqu'il décrit les changements au plan de la qualité et de la stabilité des unions à travers les trois dimensions indépendantes l'une de l'autre: les vulnérabilités personnelles, les évènements stressants et les processus adaptatifs du couple. En effet, selon ce modèle les vulnérabilités (variables personnelles), seules ou en combinaison avec les évènements stressants (facteurs externes), à travers les processus adaptatifs (variables interpersonnelles), prédiront la satisfaction conjugale (qualité de la relation), elle-même prédictrice de la stabilité de l'union du couple (poursuite ou rupture de la relation).

\section{Transition à la parentalité et Attachement}

La transition à la parentalité peut générer des bouleversements et réaménagements sur le plan individuel et dyadique (Favez, 2013). En fait, combinée à des facteurs de vulnérabilité chez les partenaires, la transition à la parentalité peut avoir des impacts sur leur fonctionnement du couple. Ces facteurs sont de type intra personnel tels que les attitudes, les caractéristiques de la personnalité, le jeune âge des conjoints (ou leur immaturité), et des facteurs de type inter personnels tel que les styles d'attachement. 
La théorie de l'attachement a été élaborée par Bowlby (1969-1982) pour étudier la relation affective entre le petit enfant et la ou les principales personnes qui en prennent soin (figures d'attachement). Il s'agit d'un mécanisme de survie qui pousse l'enfant à adopter des comportements lui permettant de se rapprocher de son donneur de soin lorsqu'une menace de danger (réelle ou imaginaire) se présente à lui afin d'aller chercher de la protection. La qualité et la consistance des soins reçus en enfance, c'est-à-dire la manière dont la ou les figures d'attachement décodent et répondent aux besoins de l'enfant contribueront au développement des modèles internes opérants à l'égard de soi et d'autrui (Ainsworth, Blehar, Waters, \& Wall, 1978). Ces modèles d'interaction appris influenceront positivement le désir de proximité à l'âge adulte ou inversement provoqueront une tendance à être anxieux, une recherche incessante d'approbation ou encore un sentiment de rejet ou d'abandon et un évitement des relations intimes.

Dans la théorie de l'attachement adulte, la typologie quadrifide proposée par Bartholomew et Horowitz (1991) est actuellement la plus utilisée par la majorité des chercheurs de la communauté scientifique. Elle identifie quatre styles d'attachement (sécurisé, craintif, préoccupé et détaché) qui découle de l'interaction entre le modèle de soi (anxiété ou peur d'être abandonné) et d'un modèle de l'autre (évitement ou peur de l'intimité).

Le style d'attachement d'un individu peut subir des changements lorsque des évènements de vie importants les confrontent comme le fait de devenir parent, et ce, autant pour la parentalité biologique et la parentalité adoptive (Fonagy, 1999). Il peut y avoir une perte momentanée, une aggravation ou encore une réparation du sentiment de sécurité interne chez les nouveaux parents (Vinay et al., 2014). Ainsi, lors de la transition à la parentalité, leurs représentations par rapport à eux-mêmes et à leur interaction avec l'entourage peuvent en être influencées (Zittoun \& Perret-Clermont, 2001 ; Santona \& Zavattini, 2005).

\section{Transition à la parentalité et Communication}

Les habilités des couples au niveau de la communication et la résolution des problèmes ont été considérées comme des indicateurs de la satisfaction conjugale au cours de la transition à la parentalité (Cox, Paley, Burchinal, \& Payne, 1999).

Certains auteurs soutiennent que la transition à la parentalité affecte la communication en diminuant les échanges positifs (Belsky \& Kelly, 1994; Cowan \& Cowan, 1994) ou en augmentant les conflits conjugaux (Belsky \& Kelly, 1994 ; Cowan \& Cowan, 1994; Shapiro, Gottman, \& Carrère, 2000) ou encore en rendant la communication plus instrumentale et moins émotionnelle; Bigras \& Paquette, 2000). D'autres auteurs (Provost \& Tremblay, 1991) trouvent que l'arrivée de l'enfant n'engendre pas de profonds 
problèmes chez les couples fonctionnels comme elle ne rapproche pas tellement les couples qui avaient des difficultés pré partum. Pour d'autres, elle peut rapprocher les couples distants (Feldman, 1971) comme elle peut créer un éloignement entre les partenaires (Cowan \& Cowan, 1994). De façon générale, les couples avec enfant ont plus tendance à rapporter des difficultés de communication avec un évitement des conflits ou des affrontements négatifs, alors que les couples sans enfants interagissent davantage positivement (Bigras \& Paquette, 2000).

\section{Transition à la parentalité et satisfaction conjugale}

Des résultats divergents ressortent quant à l'impact de la transition à la parentalité sur la satisfaction conjugale. En effet, pour certains la transition à la parentalité peut influencer négativement l'ajustement dyadique des couples en minimisant le romantisme au profit d'échanges plus axés sur les charges, les responsabilités, les obligations, les choix et les tâches (Bigras \& Paquette, 2000 ; Bodenmann, 2003). Le temps consacré à l'enfant augmente et l'attention envers le conjoint et son travail diminue. Cette situation peut provoquer chez ce dernier un sentiment de jalousie et d'être éloigné de la triade, n'ayant plus la même attention et la même affection que celles avant l'arrivée de l'enfant (Savoy, 2003). D'autres trouvent que la transition à la parentalité peut influencer positivement l'ajustement dyadique en rendant les couples plus satisfaits par rapport aux couples sans enfant, en dépit un déclin transitoire de la satisfaction conjugale peut se produire pendant les premiers mois de l'arrivée de l'enfant (Lawrence, Cobb, Rothman, Rothman, \& Bradbury, 2008 ; Tomlinson, 1996).

La présente étude vise examiner l'impact de la transition à la parentalité adoptive sur le fonctionnement conjugal chez les personnes infertiles en Tunisie. Elle s'appuie sur le modèle de fonctionnement du couple Vulnérabilité-Stress-Adaptation (VSA) développé par Karney et Bradbury (1995). Les représentations d'attachement (vulnérabilités personnelles), combinées à l'adoption (facteur de stress) seront liés à la satisfaction conjugale à travers le processus adaptatif de la communication. Chaque segment de ce modèle sera évalué dans la présente étude via la comparaison des couples infertiles devenant parents adoptifs (groupe expérimental) à des parents biologiques et des couples infertiles sans enfant (groupes contrôles). Ainsi, l'étude se propose de (1) vérifier d'abord le type d'attachement des couples infertiles avec enfant par rapport à celui des couples fertiles avec enfant et les couples infertiles sans enfant, (2) ensuite de déterminer la nature de la communication des premiers en comparaison aux deux autres groupes et enfin (3) d'identifier le niveau de la satisfaction conjugale chez les couples infertiles avec enfant par rapport à celui des deux groupes contrôles. 


\section{Méthode}

\section{Devis de la recherche}

Il s'agit d'une étude transversale présentant un devis descriptif corrélationnel qui se base sur l'approche quantitative. Elle a pour but de vérifier si les représentations d'attachement, combinées au facteur de stress (un premier facteur de stress qui est explicite et en rapport avec la présence/absence d'un enfant dans le couple et un second facteur qui est implicite et en lien avec l'infertilité), sont liés à la satisfaction conjugale au travers le processus adaptatif de la communication.

\section{Participants}

L'échantillon total est composé de 146 participants (73 couples) âgés de 23 à 60 ans et mariés depuis au moins 2 ans. Il est subdivisé en un groupe expérimental appelé "Groupe Avec Enfant Adoptif" (GAEA) composé de 30 couples (60 personnes) infertiles qui ont eu accès à une parentalité adoptive de l'Institut National de Protection de l'Enfance (INPE) et sélectionnés sur la base de leur dossier selon des critères d'inclusions (couples de nationalité tunisienne, mariés depuis au moins deux ans, dont l'un des partenaires ou les deux souffre(ent) de problèmes de fertilité, l'âge des femmes variant entre 25 et 50 ans et celui des hommes entre 25 et 60 ans et qui ont obtenu une adoption de L'INPE d'au moins un enfant) et des critères d'exclusion (célibataires, divorcés, veufs(ves), couples remariés, mariés depuis moins de deus ans, ayant dans leur histoire de couple une grossesse et/ou des enfants légitimes, épouses âgées de moins de 25 ans et plus de 50 et époux âgés de moins de 25 ans et plus de 60 ans. Le groupe contrôle choisi aléatoirement est subdivisé en deux sous-groupes : le "Groupe Avec Enfant Biologique" (GAEB) est composé de 30 couples (60 personnes) fertiles vivant une parentalité biologique. Il est sollicité de diverses façons (p. ex., par l'entremise des collègues et amis) et le "Groupe Sans Enfant" (GSE) est composé de 13 couples (26 personnes) infertiles en phase de traitement de leurs problèmes de fertilité et n'ont jamais eu d'enfant. Ils ont été sélectionnés à partir d'une population consultant au centre de procréation médicalement assistée à l'hôpital Aziza Othmana.

\section{Instruments de mesure}

En plus du questionnaire de renseignements sociodémographiques, les participants ont complété trois instruments de mesure.

Le questionnaire des Expériences Amoureuses (Experiences in

Close Relationships «ECR»; Brennan, Clark, Shaver, 1998 ; Lafontaine \& Lussier, 2003) est composé de 36 items repartis en deux dimensions : l'évitement de l'intimité (18 items) et l'anxiété d'abandon (18 items) qui sont cotées sur une échelle de 1 à 7 points (fortement en désaccord 
à fortement en accord). Lafontaine et Lussier, (2003) rapportent des coefficients de cohérence interrne élevé de 0,88 à la fois pour d'évitement de l'intimité et l'anxiété d'abandon. Dans la présente recherche, les coefficients de fidélité sont respectivement de 0,81 et de 0,79 pour les échelles de l'évitement de la proximité et de l'anxiété d'abandon.

\section{Le questionnaire des patrons de communication (Christensen, \&}

Sullaway, 1984 ; Lussier, 1995) permet d'évaluer la qualité de la communication des conjoints durant trois phases de conflits ; lorsqu'un conflit se présente (4 items), pendant que le couple en discute (18 items) et après la discussion (13 items). Il est composé de 35 items accompagnés d'une échelle de type Likert en 9 points, allant de très improbable (1) à très probable (9). Les réponses individuelles des conjoints permettent d'indiquer le niveau de probabilité d'interaction du couple lors de sa discussion d'un problème selon trois principales modes : les modes symétriques positifs (les deux partenaires expriment mutuellement leurs sentiments de manière positive : communication mutuelle, compréhension mutuelle, résolution mutuelle...), les modes symétriques négatifs (les deux partenaires expriment mutuellement leurs sentiments de manière négative ; menace mutuelle, blâme mutuel, repli mutuel...) et les modes asymétriques où il y a les jeux de rôles complémentaires et distincts dans un couple (l'un des partenaires critique pendant que l'autre se défend ; l'un des partenaires menace pendant que l'autre recule). La fidélité et la validité de ce test ont été démontrées dans plusieurs recherches comme celle de Brassard (2003) qui rapporte des coefficients oscillant entre 0,54 et 0,8. Dans la présente recherche, les coefficients alpha atteignent respectivement 0,79 (communication mutuelle), 0,70 (total demande/retrait), 0,57 (conjoint demande/répondant se retire), 0,61 (Répondant demande/ conjoint se retire), 0,66 (évitement mutuel) et 0,87 (communication négative).

L'ajustement dyadique est évaluée à l'aide de la version abrégée en quatre items (Sabourin, Valois , \& Lussier, 2005) du dyadic adjustment scale (Spanier, 1976). Cette version corrèle significativement avec la version originale de 32 items ( $r=0,94$; Sabourin et al., 2005). Elle présente une bonne validité prédictive qui a été démontrée dans une étude longitudinale de trois ans portant sur la dissolution conjugale (Sabourin et al., 2005). Dans la présente recherche, la consistance interne est de 0,77.

\section{Analyse et traitement des données}

Différentes techniques d'analyses de données ont été utilisées. Pour les données sociodémographiques, ont été utilisées une ANOVA à mesures répétées pour le facteur Âge, une ANOVA simple pour le facteur durée de vie commune, le khi deux pour le niveau scolaire, la nature de parentalité, le type 
d'infertilité et enfin le test $t$ pour la durée de parentalité. Pour la vérification des hypothèses, l'analyse utilisée est l'analyse de variance à plan mixte ou ANOVA à mesures répétées qui s'applique en cas de présence de plusieurs variables dépendantes (mesures répétées) et plusieurs variables indépendantes. Ainsi, les deux variables indépendantes sont la présence/ absence de l'enfant ou (Groupe) ; il existe par ce fait trois groupes : Groupe Avec Enfant Adoptif (GAEA), Groupe Avec Enfant Biologique (GAEB) et Groupe Sans Enfant (GSE) et le couple (formé par Homme/Femme) ou (Sexe). Les variables dépendantes sont l'attachement amoureux, la communication et la satisfaction conjugale.

Il est à signalé que dans la présente étude, l'attention est centrée sur les différences inter-sujets. La vérification des hypothèses est basée sur les comparaisons entre les trois groupes. Le «couple», facteur intra sujet s'est imposé dans les analyses. Ainsi, les comparaisons multiples ont été utilisées pour tous les facteurs significatifs intergroupes et intragroupes mais ne seront présentées et discutées que les résultats intergroupes.

\section{Résultats}

\section{Analyses statistiques descriptives}

Les analyses descriptives relatives aux informations sociodémographiques montrent une différence significative entre GAEA et les groupes contrôles concernant l'âge $(F(2,70)=9,81, \mathrm{p}<0,01)$; Le GAEA est significativement plus âgé que les deux groupes contrôles (absence de différence significative entre ces deux derniers), le nombre moyen d'années de vie commune $(F(2,70)=5,089, \mathrm{p}<0,01)$; Le GAEA rapporte un plus grand nombre moyen d'année de vie commune uniquement par rapport au GSE (la différence est aussi significative entre le GAEB et le GSE), le niveau de scolarité $\left(\chi^{2}(4)=28,73, p<0,001\right)$; Le pourcentage du niveau primaire est le plus élevée chez le GAEA $(43,33 \%)$ par rapport au GSE $(35,41)$ et le GAEB $(21,66)$. De même pour le niveau secondaire, la proportion du niveau de scolarité secondaire du GAEA $(43,33)$ semble presque égale par rapport à celle du GSE $(42,30)$ mais élevée à celle du GAEB $(29,99 \%)$ alors que le niveau de scolarité supérieur est le plus bas chez le GAEA $(13,33)$, par rapport au GSE $(30,76)$ et au GAEB $(48,33)$. Le nombre moyen d'années de parentalité $(\mathrm{t}(58)=(-5,31), \mathrm{p}<0,001)$ dans le GAEA ne dépasse pas les 5 années $(M$ $=1,97 ; E ́ T=1,159)$, alors qu'il s'étale jusqu'à 24 ans pour le GAEB $(M=8,27$; $E ́ T=6,389)$. De plus, la nature de la parentalité $\left(\chi^{2}(2)=56,12, p<0,001\right)$ est significativement différente. Tous les participants dans le GAEB ont eu accès à une parentalité biologique, tandis que dans le GAEA, 17 couples ont eu recours à une adoption et 13 couples ont fait une tutelle officieuse. Enfin, le type d'infertilité $\left(\chi^{2}(8)=76,60, \mathrm{p}<0,001\right)$ diverge entre les groupes. Le GAEA a une proportion d'infertilité masculine plus dominante $(36,66 \%)$ par 
rapport à $(23,07 \%)$ chez le GSE. Ce dernier possède des proportions d'infertilité féminine $(53,48 \%)$ et mixte $(23,07 \%)$ plus élevées que celle du GAEA qui sont respectivement de $(43,33 \%)$ et $(13,33 \%)$.

\section{Analyses statistiques inférentielles}

Il est à rappeler que cette étude est une étude inter-comparative entre trois groupes qui vise à vérifier l'impact de la transition à la parentalité sur les couples. De ce fait, seuls les résultats entre les groupes seront discutés dans la section interprétation.

L'analyse de variance à mesures répétées a été calculée pour les deux dimensions «Anxiété d'abandon» et «Evitement de proximité » du questionnaire sur l'attachement. Les résultats de l'analyse de la première dimension sont présentés dans le tableau 1.

Tableau 1 : Analyse de variance à mesures répétées des résultats des trois groupes de participants à l'échelle d' «anxiété d'abandon»

\begin{tabular}{|c|c|c|c|c|c|c|}
\hline Variables & $\begin{array}{l}\text { Source } \\
\text { variation }\end{array}$ & de & dl & $\begin{array}{l}\text { Carré } \\
\text { moyen }\end{array}$ & $F$ & $\eta^{2}$ \\
\hline \multirow{5}{*}{$\begin{array}{c}\text { Anxiété } \\
\text { D'abando } \\
\mathbf{n}\end{array}$} & Groupe & & 2 & 3,699 & $6,362 * *$ & 0,154 \\
\hline & Erreur & & 70 & 0,581 & & \\
\hline & Sexe & & 1 & 4,431 & $9,189 * *$ & 0,116 \\
\hline & $\begin{array}{l}\text { Sexe } \\
\text { Groupe }\end{array}$ & $\mathrm{X}$ & 2 & 0,641 & 1,330 & 0,037 \\
\hline & Erreur & & 70 & 0,482 & & \\
\hline
\end{tabular}

L'analyse montre que la valeur $F$ est significative pour la variable «Sexe» (les femmes ont une moyenne d'anxiété abandonnique $(4,83)$ plus élevée que celle des hommes $(4,41)$ ) et la variable «groupe » (le GAEA présente une moyenne d'anxiété d'abandon $(4,81)$ supérieure à celle du GAEB $(4,36)$ et à celle du GSE $(4,69))$.

Des comparaisons multiples (test de Bonferroni) ont été appliquées aux deux facteurs principaux «Sexe» et «Groupe» afin de mieux localiser les différences. Elles montrent pour le facteur principal «Sexe» que la moyenne d'anxiété abandonnique des femmes dans le GAEA $(5,05)$ est plus élevée que celle de leur conjoint (4,57), elle est de même chez les femmes du GAEB $(4,46)$ et celles du GSE $(4,99)$ par rapport à leur époux $(4,27)$ et $(4,39)$. Aussi, les hommes du GAEA ont une moyenne $(4,57)$ plus élevée que celle des hommes du GAEB $(4,27)$ et celle des hommes du GSE $(4,39)$. Les femmes du GAEA possèdent elles aussi une moyenne d'anxiété à l'abandon $(5,05)$ plus élevée que celle des femmes du GAEB $(4,46)$ et celle des femmes du GSE $(4,99)$.

Pour le facteur principal "Groupe », les comparaisons multiples montrent que la différence de l'anxiété d'abandon existe uniquement entre le 
GAEA et le GAEB et qu'aucune différence significative n'a été trouvée entre le GAEA et le GSE, ni entre ce dernier et le GAEB.

Les résultats de l'analyse de la deuxième dimension «Evitement de proximité » présentés dans le tableau 2 montrent un effet significatif du facteur «Sexe» qui selon lequel les femmes ont une moyenne d'évitement de proximité (2.24) plus faible que celle des hommes $(2,44)$, et un effet significatif du facteur « Groupe » qui selon lequel la moyenne d'évitement de proximité chez le GAEA $(2,09)$ est inférieure à la moyenne du GAEB $(2,43)$ et à celle du GSE $(2,50)$.

Tableau 2 : Analyse de variance à mesures répétées des résultats des trois groupes de participants à l'échelle d'évitement de proximité

\begin{tabular}{clccccc} 
Variables & $\begin{array}{l}\text { Source de } \\
\text { variation }\end{array}$ & dl & \multicolumn{2}{c}{$\begin{array}{c}\text { Carré } \\
\text { moyen }\end{array}$} & $\boldsymbol{F}$ & $\boldsymbol{\eta}^{\mathbf{2}}$ \\
\hline \begin{tabular}{c} 
Évitement $\begin{array}{c}\text { de } \\
\text { proximité }\end{array}$ \\
\cline { 2 - 7 }
\end{tabular} & Groupe & 2 & 2,287 & $5,078^{* *}$ & 0,127 \\
\cline { 2 - 6 } & Erreur & 70 & 0,450 & & \\
\cline { 2 - 7 } & Sexe & 1 & 1,229 & $5,270^{*}$ & 0,070 \\
\cline { 2 - 6 } & $\begin{array}{l}\text { Sexe } \\
\text { Groupe }\end{array}$ & 1 & 0,153 & 0,657 & 0,018 \\
\cline { 2 - 6 } & Erreur & 70 & 0,233 & & \\
\cline { 2 - 6 } & & & & & \\
\hline
\end{tabular}

Note. $\eta^{2}=$ taille de l'effet.
$* p<0,05 . * * p<0,01 . * * * p<0,001$.

Les comparaisons multiples (test de Bonferroni) appliquées aux deux facteurs principaux «Sexe » et « Groupe », montrent pour le facteur principal «Sexe» que la différence entre les hommes et les femmes concernant l'évitement de proximité existe dans les trois groupes. En effet, la moyenne d'évitement de proximité des hommes dans le GAEA $(2,24)$ est plus élevée que celle de leur conjointe $(1,95)$, elle est de même chez les hommes du GAEB $(2,57)$ et les hommes du GSE $(2,52)$ par rapport à leur épouse $(2,30)$ et $(2,48)$. Chez les hommes, la moyenne d'évitement de proximité la plus élevée est celle du GAEB $(2,57)$, puis celle du GSE $(2,52)$ et enfin celle du GAEA $(2,24)$. Chez les femmes, la moyenne la plus élevée est la moyenne du GSE $(2,48)$ puis celle du GAEB $(2,30)$ et enfin celle du GAEA $(1,95)$.

Pour le facteur principal "Groupe», les comparaisons multiples montrent que la différence de l'évitement de proximité existe entre le GAEA et le GAEB aussi bien qu'entre le GAEA et le GSE alors qu'aucune différence significative n'a été trouvée entre le GAEB et le GSE. Cette différence est plus importante entre les femmes du GAEA et les femmes du GSE qu'entre les premières et celles du GAEB. Elle est aussi plus importante entre les hommes du GAEA et les hommes du GAEB qu'entre les premiers et ceux du GSE.

Bref, pour l'effet « Groupe », la différence d'anxiété d'abandon n'est significative qu'entre le GAEA et le GAEB; le premier présente plus d'anxiété d'abandon que le deuxième. La différence d'évitement de proximité est significative entre le GAEA et les deux groupes contrôles ; Le premier 
présente moins d'évitement de proximité que le GAEB et le GSE. En somme, la première hypothèse qui stipule que le GAEA présente une plus faible anxiété d'abandon et un plus faible évitement de l'intimité par rapport aux deux groupes contrôles est partiellement confirmée. En effet, bien que les couples infertiles qui ont eu accès à une parentalité adoptive semblent éviter moins la proximité que les couples des deux groupes contrôles, ils sont plus anxieux face à l'abandon que le GAEB et ne semblent pas avoir de différence avec le GSE.

Pour la deuxième hypothèse, trois de six dimensions du questionnaire de patron de communication ont été retenues pour vérifier trois comportements ; le comportement de la communication mutuelle à travers la dimension «Communication Mutuelle », le comportement de demande-retrait via les dimensions «Total Demande-Retrait» et enfin le comportement d'évitement grâce à la dimension «Evitement Mutuel». Les résultats de l'analyse des deux premières dimensions sont présentés dans les tableaux 3 et 4.

Tableau 3 : Analyse de variance à mesures répétées des résultats des trois groupes de participants à l'échelle de « Communication Mutuelle».

\begin{tabular}{|c|c|c|c|c|c|}
\hline Variables & $\begin{array}{l}\text { Source } \\
\text { de } \\
\text { variation }\end{array}$ & d & $\begin{array}{l}\text { Carré } \\
\text { moyen }\end{array}$ & $F$ & $\eta^{2}$ \\
\hline \multirow[t]{5}{*}{$\begin{array}{r}\text { Communic } \\
\text { ation Mutuelle }\end{array}$} & Groupe & 2 & $\begin{array}{l}336,20 \\
9\end{array}$ & $\begin{array}{l}4,87 \\
7 *\end{array}$ & $\begin{array}{l}0,12 \\
2\end{array}$ \\
\hline & Erreur & $0^{7}$ & 68,937 & & \\
\hline & Sexe & 1 & 11,802 & $\begin{array}{l}0,54 \\
0\end{array}$ & $\begin{array}{l}0,00 \\
8\end{array}$ \\
\hline & $\begin{array}{cc}\text { Sexe } & X \\
\text { Groupe } & \\
\end{array}$ & 2 & 43,621 & $\begin{array}{l}1,99 \\
5\end{array}$ & $\begin{array}{l}0,05 \\
4\end{array}$ \\
\hline & Erreur & $0^{7}$ & 21,861 & & \\
\hline
\end{tabular}

Note. $\eta^{2}=$ taille de l'effet.

$* \mathrm{p}<0,05 . * * \mathrm{p}<0,01 . * * * \mathrm{p}<0,001$.

Tableau 4 : Analyse de variance à mesures répétées des résultats des trois groupes de participants à l'échelle « Total Demande-Retrait».

\begin{tabular}{clccccc} 
Variables & $\begin{array}{l}\text { Source de } \\
\text { variation }\end{array}$ & $\mathbf{d l}$ & $\begin{array}{c}\text { Carré } \\
\text { moyen }\end{array}$ & $\boldsymbol{F}$ & $\boldsymbol{\eta}^{2}$ \\
\hline $\begin{array}{c}\text { Total } \\
\text { Demande- } \\
\text { Retrait }\end{array}$ & Groupe & 2 & 295,189 & $4,137^{*}$ & 0,106 \\
\cline { 2 - 6 } & Erreur & 70 & 71,346 & & \\
\cline { 2 - 6 } & Sexe & 1 & 8,800 & 0,529 & 0,07 \\
\cline { 2 - 6 } & $\begin{array}{l}\text { Sexe } \\
\text { Groupe }\end{array}$ & 2 & 13,682 & 0,822 & 0,023 \\
& Erreur & 70 & 16,651 & & \\
\cline { 2 - 6 } & & & & & \\
\end{tabular}

Note. $\eta^{2}=$ taille de l'effet.

$* \mathrm{p}<0,05 . * * \mathrm{p}<0,01 . * * * \mathrm{p}<0,001$ 
L'analyse ne montre pas d'effet significatif du facteur « Sexe», ni de l'interaction «Sexe» $X$ «Groupe» pour les trois dimensions. Pour le facteur «Groupe», les résultats montrent, pour les deux dimensions «Communication Mutuelle » et «Total Demande-Retrait», une différence significative uniquement entre le GAEA et le GSE (absence de différence entre le GAEA et le GAEB et entre ce dernier et le GSE) alors que pour la dimension «Évitement Mutuel», aucune différence significative n'a été indiquée entre les trois groupes.

Un test de comparaison multiple (Bonferroni) a été appliqué au facteur «Groupe » pour les deux premières dimensions. Il s'est avéré que l'effet « Groupe » est dû au fait que le GAEA rapporte davantage de communication mutuelle que le GSE $(38,73$ et 32,95 respectivement, $\mathrm{p}<0,05)$ et plus de comportement demande-retrait respectivement $(38,63)$ et $(33,65)$.

Ainsi, la deuxième hypothèse qui stipule que le GAEA a moins de difficulté de communication que les deux groupes contrôles, autrement dit, il a plus de communication mutuelle et moins de comportements de demanderetrait et d'évitement que les deux autres groupes, est partiellement confirmée. Les personnes du GAEA utilisent significativement plus la communication mutuelle mais uniquement par rapport au GSE. Toutefois, elles ne semblent pas posséder moins de comportements «Demande-Retrait», au contraire, elles ont significativement davantage ces comportements par rapport au GSE. Enfin, elles ont des comportements d'évitement mutuel qui ne diffèrent pas de ceux des groupes contrôles.

Les analyses pour la troisième hypothèse qui stipule que le GAEA a une satisfaction conjugale supérieure à celle des deux groupes contrôles figurent dans le tableau 5 et montrent des effets significatifs du « Sexe», du «Groupe » et de l'interaction entre « Sexe » et « Groupe » entre le GAEA et les deux groupes contrôles (aucune différence significative n'existe entre le GAEB et le GSE).

Tableau 5 : Analyse de variance à mesures répétées des résultats des trois groupes de participants à l'échelle d'ajustement dyadique forme abrégée (DAS)

\begin{tabular}{|c|c|c|c|c|c|}
\hline Variable & $\begin{array}{l}\text { Source de } \\
\text { variation }\end{array}$ & dl & $\begin{array}{l}\text { Carré } \\
\text { moyen }\end{array}$ & $F$ & $\eta^{2}$ \\
\hline \multirow{5}{*}{$\begin{array}{l}\text { Ajustement } \\
\text { dyadique }\end{array}$} & Groupe & 2 & 63,126 & 7,436 *** & 0,175 \\
\hline & Erreur & 70 & 8,490 & & \\
\hline & Sexe & 1 & 11,443 & $5,137 *$ & 0,068 \\
\hline & Sexe X Groupe & 2 & 10,474 & $4,702 * *$ & 0,118 \\
\hline & Erreur & 70 & 2,228 & & \\
\hline
\end{tabular}

Afin de localiser les différences pour chaque facteur, le test de Bonferroni a été appliqué. 
L'effet significatif « Sexe » indique qu'il existe une différence entre la moyenne d'ajustement dyadique des hommes par rapport à celle des femmes; la première $(18,17)$ est plus élevée que la deuxième $(17,57)$. La moyenne des hommes du GAEA $(19,03)$ est légèrement faible à celle de leur épouse $(19,33)$. La moyenne des hommes du GAEB $(17,73)$ est légèrement élevée par rapport à la moyenne de leur femme $(17,46)$ et la moyenne des hommes du GSE $(17,76)$ est plus élevée que celle de leur partenaire $(15,92)$.

L'effet significatif du "Groupe" indique que le GAEA possède une satisfaction conjugale $(19,18)$ plus élevée à celle du GAEB $(17,59)$ et celle du GSE $(16,84)$ (alors qu'aucune différence significative n'existe entre le GAEB et le GSE).

L'effet d'interaction significatif entre « Sexe » et « Groupe » indique que la différence entre le GAEA et les deux groupes contrôles est très forte chez les femmes que chez les hommes; les femmes du GAEA présentent une satisfaction conjugale plus importante par rapport aux femmes du GAEB et davantage plus importante par rapport au GSE. Par contre, les hommes du GAEA possèdent une satisfaction conjugale plus élevée que celle du GAEB et du GSE. Ces deux derniers ont une satisfaction conjugale presque identique.

La troisième hypothèse est confirmée. Les couples avec enfant adoptif sont plus satisfaits que les couples avec enfant biologique et les couples sans enfant.

\section{Discussion}

La présente recherche visait à examiner l'impact de la transition à la parentalité adoptive sur le fonctionnement conjugal chez les personnes infertiles. Ses objectifs étaient de :

1. vérifier le type d'attachement des couples infertiles avec enfant par rapport à celui des couples fertiles avec enfant et les couples infertiles sans enfant,

2. déterminer la nature de la communication des premiers en comparaison aux deux autres groupes,

3. d'identifier le niveau de la satisfaction conjugale chez les couples infertiles avec enfant par rapport à celui des deux groupes contrôles.

Les résultats obtenus montrent que les personnes vivant une transition à la parentalité adoptive diffèrent sur plusieurs variables en ce qui concerne leurs données sociodémographiques. D'abord, l'âge est assez avancé chez le GAEA par rapport aux deux groupes contrôles. Ce résultat confirme celui trouvé par Laaribi et al. (2009) dans une étude tunisienne sur le profil psychosocial de la famille adoptive en consultation de pédopsychiatrie selon lequel l'âge moyen des pères adoptifs était de 45 ans au moment de l'adoption (avec des extrêmes allant de 30 ans à 60 ans) et que celui des mères était de 
38 ans (avec des extrêmes allant de 24 ans à 58 ans). Ensuite, le niveau scolaire primaire est le plus dominant chez le GAEA avec un pourcentage élevé (50\%) chez les femmes alors que pour les hommes, le niveau primaire existe avec une forte intensité mais le niveau secondaire est encore plus élevé avec une proportion de $56,66 \%$ des cas. Ceci ne rejoint pas le résultat trouvé par Laaribi et al. (2009) qui montre que le niveau scolaire de type primaire est prédominant chez les deux partenaires avec un pourcentage de $54,5 \%$ des cas pour les pères et $40,9 \%$ des cas pour les mères. Enfin, le niveau socioéconomique et la profession des participants des trois groupes n'ont pas été pris en considération parce que la majorité des personnes des deux groupes contrôles n'ont pas divulgué cette information et, par conséquent, la comparaison entre les groupes ne pourrait être réalisable. Toutefois, les données socio-économiques du GAEA montrent que plus de la moitié de cet échantillon $(78,33 \%, \mathrm{n}=47)$ possède un revenu mensuel net situé entre 300 DT et 2000 DT. Une grande proportion (43,33\%) de l'échantillon, constituée uniquement de femmes n'ont aucun revenu. Par conséquent, $56,66 \%$ des femmes et $100 \%$ des hommes possèdent un revenu mensuel. Ceci confirme le résultat trouvé par Laaribi et al. (2009) concernant une majorité (59\%) des hommes qui sont des journaliers et infirme celui concernant la majorité des femmes $(59,1 \%)$ est sans profession.

En ce qui a trait à la première hypothèse, il était prévu que le GAEA présente une faible anxiété et un faible évitement, autrement dit, un type d'attachement sécurisant, alors que les résultats mettent en relief un évitement faible et une anxiété élevée qui s'apparentent à un type d'attachement insécurisant. Ce résultat concorde avec celui de l'étude de Vinay et al., (2014) qui démontre que l'arrivée de l'enfant marque un changement dans le style d'attachement des parents, elle déstabilise le sentiment de sécurité interne qui fournit un modèle d'attachement sécurisé et elle les dirige vers des modèles d'attachement davantage insécurisés.

L'attachement insécurisant est observé dans les trois groupes avec d'un côté une forte intensité chez le GAEA par rapport aux GAEB; les différences sont significatives entre eux pour les deux dimensions avec plus d'anxiété d'abandon et moins d'évitement de proximité chez le premier groupe et d'un autre côté une faible intensité chez le GAEA par rapport au GSE. La moyenne d'anxiété abandonnique du premier ne diffère pas de celle du GSE mais la moyenne d'évitement de proximité est plus faible chez le GAEA par rapport au GSE.

Il est possible que les personnes du GAEA veulent plus d'indépendance, essaient de réduire leur peur d'être abandonnées et leur sentiment de ne plus être aimées. Il se peut aussi qu'elles se sont tournées vers l'adoption parce qu'elles ne supportent plus la « solitude » et ont besoin de plus d'attention. Devenus parents, ces couples sont plus sensibles à leur 
nouveau statut et leur nouvelles tâches. Selon la littérature, les parents adoptifs élaborent des représentations autour de leur rôle parental et du lien parentenfant en référence à leurs propres modèles relationnels de l'enfance. Ils rapportent des thèmes de «proximité relationnelle» ou «d'intimité »par rapport au thème de «disponibilité » chez les parents biologiques (Fonagy, 1999).

Pour la deuxième hypothèse, contrairement à ce qui était attendu, les résultats montrent que le GAEA utilise plus de communication mutuelle et des comportements de demande-retrait» que le GSE, mais cette différence ne ressort pas avec le GAEB (ni entre ce dernier et le GSE).

La communication mutuelle chez les couples adoptifs ne semble pas être altérée par la transition à la parentalité contrairement aux résultats trouvés par des auteurs (Belsky \& Kelly, 1994) qui indiquent que devenir parent diminue les interactions positifs entre les partenaires. Les échanges des parents adoptifs ne sont pas affaiblis après l'arrivée de l'enfant mais leurs comportements de demande-retrait se trouvent plus élevés par rapport à ceux des couples sans enfant. Des auteurs (Belsky \& Kelly, 1994 ; Shapiro et al.,2000) mentionnent que l'arrivée de l'enfant peut produire des problèmes de communication liés à une augmentation des conflits conjugaux et une baisse de satisfaction.

Les couples du GAEA ne diffèrent pas des couples des deux groupes contrôles sur le plan d'évitement mutuel contrairement à ce qui était montré dans la documentation (Jordan \& Revenson, 1999); les personnes infertiles en traitement utilisent plusieurs stratégies d'adaptation et davantage l'évitement que les couples fertiles.

Bref, la communication chez le GAEA semble parfois efficace et parfois déficiente parce que le patron de communication utilisé regroupe à la fois un mode de communication symétrique positif (communication mutuelle) et un mode asymétrique (l'utilisation indifférenciée du patron demande-retrait à l'intérieur du couple, telle que perçue par le répondant).

Les analyses dans la troisième hypothèse montrent que les couples avec enfant adoptif sont plus satisfaits que les couples avec enfant biologique et les couples sans enfant. Ce résultat rejoint celui trouvé par Galhardo, Cunha et Pinto-Gouveia (2011) qui montre que l'ajustement dyadique est plus élevé chez les couples candidats à l'adoption par rapport aux couples infertiles et les couples fertiles. La différence de l'ajustement dyadique est plus importante entre le GAEA et le GSE qu'entre le premier et le GAEB. Ce résultat concorde avec celui trouvé par Weaver, Clifford, Douglas et Robinson (1997) qui ne montre pas de différence significative entre les couples infertiles qui ont échoué la fécondation in vitro (FIV) ou le transfert intrafallopien des gamètes (TIFG) et le groupe témoin qui a réussi l'un des deux traitements. 
Le GAEA semble plus satisfait que le GAEB. Le moment de devenir parent semble jouer un rôle dans la satisfaction conjugale en dépit de la durée de parentalité. Les couples infertiles sont devenus parents après des années de traitements et d'attente et la moyenne de la durée de la parentalité adoptive ne dépasse pas 1,97 an en comparaison aux couples fertiles qui ont eu leur premier enfant dans les premières années de mariage et vivent une parentalité depuis en moyenne 8,27 ans. Ce résultat concorde avec celui trouvé par Lemieux en 2008 qui montre qu'une parentalité très récente (survenant entre 2 ans à 6 ans de formation du couple) est vécue avec beaucoup de stress et avec un rythme accéléré par rapport à une parentalité qui vient après plus de 7 ans.

\section{Conclusion}

La présente recherche offre une meilleure compréhension des changements au sein du couple. Elle a l'avantage non seulement d'apporter de nouvelles connaissances et des données auprès d'une population spécifique constituée de couples infertiles qui ont eu recours à une parentalité adoptive mais aussi de disposer d'un échantillon de couples varié (couples fertiles avec enfants, couples infertiles avec enfants et couples infertiles sans enfants) afin de contraster différentes situations comme le fait d'avoir un enfant ou non mais en plus de pouvoir comparer la présence d'un enfant chez des parents adoptifs à celle chez des parents biologiques. Elle permet de documenter le profil psychosocial de la famille adoptive en Tunisie à travers les différentes caractéristiques sociodémographiques des parents adoptifs et ouvre plusieurs pistes d'investigation pour les futures études qui viseront à fournir de nouvelles réflexions et à mieux identifier les problématiques associées au fonctionnement conjugal des couples aux prises avec des problèmes de fertilité.

Une recherche longitudinale pour déterminer l'évolution des représentations d'attachement, de l'ajustement dyadique et de la communication chez les personnes infertiles qui ont eu accès à une parentalité adoptive serait intéressante. De même, des analyses qualitatives basées sur des entrevues cliniques pourraient être complémentaires aux analyses quantitatives et apporter de nouveaux éléments de compréhension d'où l'importance d'enrichir le travail par les entretiens cliniques et d'autres types d'outils d'investigation (p. ex., les méthodes projectives). Par ailleurs, le terme adoption dans cette recherche a regroupé l'adoption et la tutelle officieuse. Les couples qui ont accueilli un enfant dans le cadre de l'adoption pourraient avoir des résultats différents de ceux qui ont eu recours à la tutelle officieuse. Il serait intéressant dans les futures recherches de faire des comparaisons entre les deux types de parentalité adoptives. Il serait aussi pertinent de refaire cette étude avec des couples infertiles qui ont terminé le traitement de leurs 
problèmes de fertilité et d'approfondir les recherches sur les caractéristiques de nouveaux types de parentalité à travers une étude comparative entre la parentalité biologique, la parentalité adoptive et la «parentalité médicalement assistée ».

\section{Déclaration d'intérêt}

L'auteure déclare n'avoir aucun lien d'intérêt en relation avec cet article.

\section{References:}

1. Ainsworth, M. D. S., Blehar, M. C., Waters, E., \& Wall, S. (1978). Patterns of attachment: A psychological study of the Strange Situation. Hillsdale (Wiley), New York.

2. Barraud, E. (2008). Adoption et Kafala dans l'espace migratoire franco-maghrébin. Dossier : La fabrique de la mémoire (459-468).

3. Bartholomew, K., \& Horowitz, L. M. (1991). Attachment styles among young adults: A test of a four-category model. Journal of Personality and Social Psychology, 61, 226-244.

4. Brassard, A. (2003). Pratique d'activités physiques et de loisir, communication et ajustement dyadique. Mémoire. Trois-Rivières : Université du Québec à Trois-Rivières, 123 p.

5. Belsky, J., \& Kelly, J. (1994). The transition to parenthood: How a first child changes a marriage. Why some couples grow closer and others apart. New-York: Dell.

6. Bigras, M., \& Paquette, D. (2000). L'interdépendance entre les soussystèmes conjugal et parental: une analyse personne- processuscontexte. Psicologia: Teoria e Pesquisa, 16 (2), 91-102.

7. Bodenmann, G. (2003). Une vie de couple heureuse. Paris : Odile Jacob.

8. Brennan, K.A., Clark, C.L., \& Shaver, P. R. (1998). Self-report measurement of adult romantic attachment: An integrative overview. In J.A. Simpson \& W.S. Rholes (Eds.), Attachment theory and close relationship (pp.46-76). New York : Guilford Press.

9. Cox, M.J, Paley, B., Burchinal, M., \& Payne, C.C. (1999). Marital perceptions and interactions across the transition to parenthood. Journal of Marriage and the Family, 61, 611-625.

10. Cowan, C.P., \& Cowan, P.A. (1994). $1+1=3$ : quand le couple devient famille. Ce changement fondamental dans la vie d'un couple. Paris : J'ai Lu.

11. Favez, N. (2013). La transition à la parentalité et les réaménagements de la relation de couple. Eres/Dialogue, 199, 73-83. 
12. Feldman, H. (1971). The effects of children on the family. In A. Michel, (Éd.), Family issues of employed women in Europe and America. E.J. Brill, Leiden, The Netherlands, 107-125.

13. Fonagy, P. (1999). Psychoanalytic theory from the viewpoint of attachment theory and research. In J. Cassidy, \& P. Shaver (Éds.), Handbook of attachment theory and research (pp. 595-624). New York: Guilford Press.

14. Frascarolo, F., Darwiche, J., \& Favez, N. (2009). Couple conjugal et couple co-parental: quelle articulation lors de la transition à la parentalité? De Boeck Supérieur: Cahiers critiques de thérapie familiale et de pratiques de réseaux, 42, 207-229.

15. Galhardo, A., Cunha, M., \& Pinto-Gouveia, J. (2011). Aspects psychologiques des couples infertiles. Sexologies, 20, 255-260.

16. Jordan, C., \& Revenson, T.A. (1999). Gender differences in coping with infertility: A meta-analysis. Journal of Behavioral medecine, 22(4), 341-358.

17. Karney, B.R, \& Bradbury, T.N. (1995). The longitudinal course of marital quality and stability: A review of theory, method and research. Psychological Bulletin, 118 (1), 3- 34.

18. Laaribi, A., Moalla, Y., Hadj Kacem, I., Ayadi, H., Walha, A., \& Ghribi, F. (2009). Profil psychosocial de la famille adoptive en consultation de pédopsychiatrie. Arabpsynet E. Journal, 21-22, 96-99.

19. Lafontaine, M.-F., \& Lussier, Y. (2003). Structure bidimensionnelle de l'attachement amoureux : anxiété face à l'abandon et évitement de l'intimité. Revue canadienne des sciences du comportement, 35(1), 5660.

20. Lawrence, E., Cobb, R.J., Rothman, A.D., Rothman, M.T., \& Bradbury, T.N. (2008). Marital satisfaction across the transition to parenthood. Journal of Family Psychology, 22(1), 41-50.

21. Provost, M-A., \& Tremblay, S. (1991). Le nouveau-né et le couple : Adversaires ou partenaires ? Santé Mental au Québec, 16(1), 235-250.

22. Sabourin, S., Valois, P., \& Lussier, Y. (2005). Development and validation of a brief version of the Dyadic Adjustment Scale with a nonparametric item analysis model. Psychological Assessment, 17(1), $15-27$.

23. Santona, A., \& Zavattini, G. C. (2005). Partnering and parenting expectations in adoptive couples. Sexual and Relationship Therapy, 20(3), 309-322.

24. Savoy, J. Y. (2003). Couple et aventure : le couple et l'arrivée de l'enfant. Lausanne, Suisse : Editions La Passerelle.

25. Shapiro, A.F., Gottman, J. M., \& Carrère, S. (2000). The baby and the marriage: Identifying factors that buffer against decline in marital 
satisfaction after the first baby arrives. Journal of Family Psychology, 14(1), 59-70.

26. Tomlinson, P.S. (1996). Marital relationship change in the transition to parenthood: A reexamination as interpreted through transition theory. Journal of Family Nursing, 2 (3), 286-305.

27. Vinay, A., Brenot-Bergeret, M., Rosenblum, O., \& Genty, M. (2014). Réflexions autour du processus de parentalité : quelles étapes et quelles spécificités dans l'adoption? Pratiques Psychologiques, 20, 21-37.

28. Weaver, S.M., Clifford, E., Douglas, M.H., \& Robinson, J. (1997). Psychosial adjustment to unsuccessful IVF and GIFT treatment. Patient Education and Counseling, 31, 7-18.

29. Zeghiche, S. \& deMontigny, F., (2014). Transition à la parentalité et son impact sur la relation conjugale : Une analyse des travaux de Dr Nicolas Favez, Gatineau, QC : CERIF/UQO.

30. Zittoun, T., \& Perret-Clermont, A-N. (2001). Contributions à une psychologie de la transition. Congrès international de la Société suisse pour la recherche en éducation (SSRE), 1-11. 